\title{
EFFECTIVENESS OF CONVENTIONAL PHOTOTHERAPY VERSUS SUPER LIGHT- EMITTING DIODES PHOTOTHERAPY IN NEONATAL HYPERBILIRUBINEMIA
}

S. Ngerncham ${ }^{1}$, K. Jirapaet ${ }^{1}$, R. Suwanchai ${ }^{2}$, R. Chaweerat $^{2}$, P. Wongsiridej ${ }^{1}$, T. Kolatat $^{1}$

${ }^{I}$ Division of Neonatology, Department of Pediatrics, Faculty of Medicine Siriraj Hospital, Mahidol

University, ${ }^{2}$ Pediatric Nursing Division, Nursing Department, Siriraj Hospital, Bangkok, Thailand

Background: Neonatal hyperbilirubinemia is very common. Phototherapy has been used for decades to prevent severe hyperbilirubinemia, which can cause kernicterus. We compare the effectiveness of two phototherapy devices in reducing plasma bilirubin and duration of phototherapy in non-severe hyperbilirubinemia.

Materials and methods: This was an open-label randomized controlled trial. Forty healthy infants aged between 1 and 5 days with non-severe hyperbilirubinemia, but to the level requiring phototherapy, were recruited. The phototherapy unit used in the "blue-light" group was the Siriraj Phototherapy Lamp with 6 special blue fluorescent tubes. The phototherapy unit used in the "light-emitting diodes (LEDs)" group was the Bilitron 3006 with 5 super LEDs. The distance between both devices and the infants was fixed at $30 \mathrm{~cm}$.

Results: There were 20 infants in each group. Mean gestational age was $38.0 \pm 1.5$ weeks. Baseline clinical characteristics of infants in both groups were comparable. Median rate of plasma bilirubin decreasing during phototherapy in "blue light" was significantly less than in "LEDs" group. [median (25\%, 75\%tile) 0.16 $(0.09,0.25)$ and $0.10(0.02,0.17) \mathrm{mg} / \mathrm{dL} / \mathrm{hr}$, respectively; $p=0.03]$. Duration of phototherapy with "blue light" was not statistically different from phototherapy with "LEDs" [median (25\%, 75\%tile) 23.0 (19.0, $30.8)$ and $30.0(22.3,40.3) \mathrm{hr}$, respectively; $p=0.11]$.

Conclusion: A locally invented phototherapy device with special blue fluorescents tubes can be more effective than the more expensive commercial super LEDs phototherapy device in decreasing plasma bilirubin. 